\title{
Kinetics of pressure-induced nanocrystal superlattice formation
}

\author{
Felix Lehmkühler, ${ }^{* a, b}$ Martin A. Schroer, ${ }^{c}$ Verena Markmann, ${ }^{a}$ Lara Frenzel, ${ }^{a, b}$ Johannes \\ Möller, ${ }^{d}$ Holger Lange, ${ }^{b, e}$ Gerhard Grübel, ${ }^{a, b}$ and Florian Schulz ${ }^{b, e}$
}

Received Date

Accepted Date

DOI: 00.0000/xxxxxxxxxx

Colloidal nanocrystals (NC) are known to self-organize into superlattices that promise many applications ranging from medicine to optoelectronics. Recently, the formation of high-quality PEGylated gold NC was reported at high hydrostatic pressure and high salt concentrations. Here, we study the formation kinetics of these superlattices after pressure jumps beyond their crystallisation pressure by means of small-angle X-ray scattering with few ms experimental resolution. The timescale of NC formation was found to be reduced the larger the width of the pressure jump. This is connected to an increase of crystal quality, i.e., the faster the NC superlattice forms, the better the crystal quality. In contrast to the formation kinetics, the melting of the NC superlattice is approximately one order of magnitude slower and shows linear kinetics.

Highly ordered 3D assemblies of nanocrystals (NC), so called supercrystals or superlattices, attract ongoing research interest because they allow for designing new materials with properties tunable via their geometry and composition ${ }^{1+1+5}$. In the case of gold nanocrystals (AuNC), especially their plasmonic properties are of interest $\frac{677}{6}$. In AuNC superstructures, interparticle plasmonic coupling results in new emerging modes and strongly enhanced electric fields on the nanoscale that can be utilized for high performance enhanced spectroscopies and optical metamaterials 7 . Plasmonic properties strongly depend on the geometry of the superstructures. A well defined, crystalline geometry results in well defined optical properties (e.g., sharper absorption

\footnotetext{
a Deutsches Elektronen-Synchrotron DESY, Notkestr. 85, 22607 Hamburg, Germarny, E-mail: felix.lehmkuehler@desy.de

${ }^{b}$ The Hamburg Centre for Ultrafast Imaging, Luruper Chaussee 149, 22761 Hamburg, Germany.

${ }^{c}$ European Molecular Biology Laboratory EMBL c/o DESY, Notkestrasse 85, 22607 Hamburg, Germany.

${ }^{d}$ European XFEL GmbH, Holzkoppel 4, 22869 Schenefeld, Germany.

${ }^{e}$ Institute of Physical Chemistry, University of Hamburg, Grindelallee 117, 20146 Hamburg, Germany.
}

peaks compared to a less ordered system) and light-matter coupling strengths, field enhancements and energies of plasmonic modes are dictated by the crystal structure and lattice constant among others ${ }^{677}$. In consequence, a proper understanding of superlattice formation is essential to control such parameters.

The most common way of producing such superlattices is the self-organisation from nanocrystal suspensions ${ }^{8}$ 12. This process is usually based on solvent evaporation, precipitation or the addition of a non-solvent ${ }^{111}$ and consequently has to be slow to obtain high-quality superlattices. The in situ NC self-assembly processes are usually studied by X-ray scattering methods at synchrotron radiation sources, allowing for different experimental environments and geometries $\frac{13-18}{18}$. However, as many studies focus on the structure and structural transitions little is known about the kinetics of the superlattice formation $19-21$.

We have recently demonstrated that pressure can trigger the assembly of PEGylated AuNC into highly ordered superlattices in solution ${ }^{22}$. While the majority of high-pressure experiments on NC assemblies investigate pressure effects on pre-ordered assemblies $\frac{23}{2}$, we observed the formation of superlattices from a liquid state. A striking feature was that superlattices were formed fast, within seconds, and reversibly, indicating a phase transition. This could be explained by the combined effect of salt and pressure on the PEG ligand shells of the AuNC. Here, we study the kinetics of superlattice formation in more detail. To this end we performed different pressure jumps around the transition pressure and monitored the response of the system with time-resolved small-angle X-ray scattering (SAXS). Experiments at modern synchroton radiation sources provide high-quality SAXS data of soft matter samples with time resolution in the (sub-)ms regime thus allowing to monitor the structure evolution and kinetics of superlattice formation and dissolution in situ. We find a clear dependence, structurally and kinetically, on the magnitude $\Delta p$ of the pressure jump. The formation kinetics of the superlattice exhibits an exponential trace with a growth rate determined by the increase of attractive interparticle interaction due to deswelling of the PEG shell. In 
contrast, the dissolution of the superlattices upon reducing the pressure follows an initial linear kinetics.

\section{Experimental}

\section{Sample preparation}

We employed gold nanocrystals with a radius of about $6 \mathrm{~nm}$ and with a low dispersity of less than $7 \%$ that were prepared following earlier studies $24 \mid 25$. The nanocrystals were coated with $\alpha$-methoxypoly(ethylene glycol)- $\omega$-(11mercaptoundecanoate) (PEGMUA) ligands with $M \approx 5000 \mathrm{~g} / \mathrm{mol}$. The NC were dispersed in an aqueous solution with $2 \mathrm{M} \mathrm{RbCl}$. The particle concentration was $500 \mathrm{nM}$ corresponding to a volume fraction of about 1 vol\% AuNC. This system is known to selfassemble after solvent evaporation $26[27$ and is supposed to form a superlattice around $p \approx 3000$ bar 22 .

\section{X-ray scattering experiment}

The SAXS experiment was conducted at beamline I22, Diamond Light Source (Didcot, UK) 28 . We used a hydrostatic high-pressure set-up 29 which is sealed with two diamond windows. This environment was designed for performing pressure jumps within $t_{j} \approx 5 \mathrm{~ms}$. The X-ray energy was set to $18 \mathrm{keV}$, corresponding to a wave-length of $\lambda=0.68 \AA$, allowing to penetrate the windows of the pressure cell. The sample suspensions were filled into polycarbonate capillaries, sealed and placed into the pressure chamber before the measurements. SAXS patterns were recorded by a PILATUS 3-2M detector $6.7 \mathrm{~m}$ downstream the sample. The beam size (FWHM) was $140 \mu \mathrm{m} \times 230 \mu \mathrm{m}$ (v x h). First, we carefully checked for radiation damage of the sample by using different absorbers attenuating the beam intensity on the sample. Second, we verified the crystallisation pressure of the suspension by taking SAXS patterns at pressures above 2000 bar in steps of 100 bar. We observed crystallisation at a pressure $p_{c}$ between 2900 bar and 3000 bar which coincides with our previous results for the salt and particle concentration used here 22 . For studying structural variations after a pressure jump, we first set the pressure below the crystallisation pressure to $p_{0}$. We measured a sequence of SAXS patterns of $30 \mathrm{~s}$ duration with the maximum repetition rate of the detector of $250 \mathrm{~Hz}$, i.e. $t_{\min }=4 \mathrm{~ms}$ resolution. After $t=t_{j}=1 \mathrm{~s}$ (250 SAXS patterns), the pressure jump took place to the final pressure $p_{f}$. This procedure allows us to compare the sample before and after the pressure jump with decent counting statistics. In the following, data was averaged over five frames to improve the statistics, leading to an effective time resolution of $t_{\mathrm{min}, \mathrm{eff}}=20 \mathrm{~ms}$.

\section{Results}

In SAXS experiments on spherical particles, the intensity is given by $I(q) \propto P(q) S(q)$, with the wave vector transfer $q=$ $4 \pi \sin (\theta / 2) / \lambda$ and scattering angle $\theta$. The particle form factor $P(q)$ contains information about the particle size, dispersity and shape and can be measured using a dilute dispersion. The structure factor $S(q)$ as the Fourier transform of the radial distribution function reflects interparticle interactions, e.g., Bragg reflections in case of crystalline samples. SAXS patterns taken at 2900 bar a

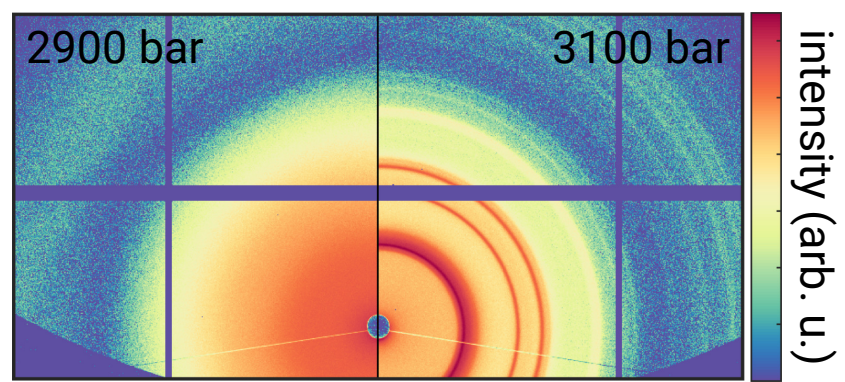

b

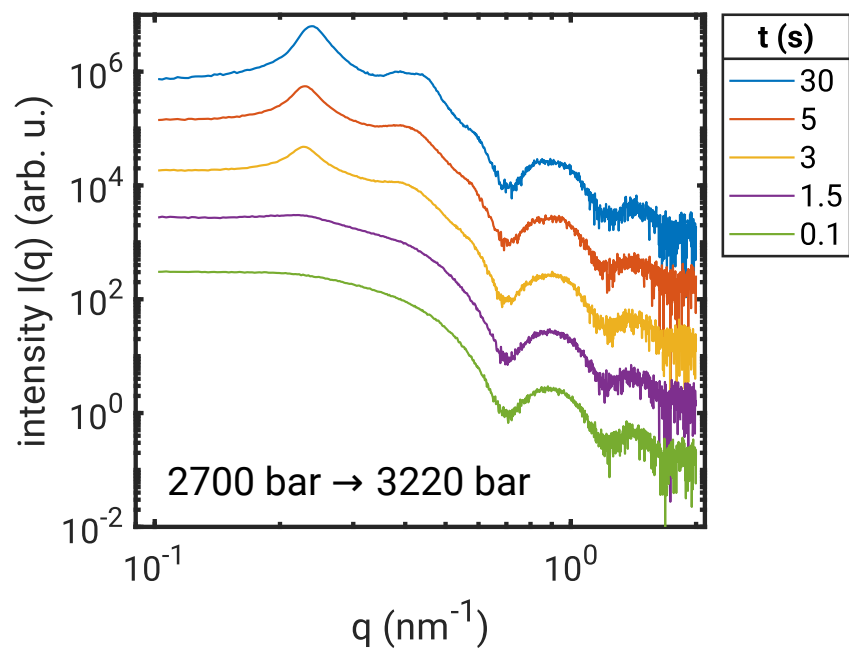

Fig. 1 (a) Two dimensional SAXS patterns from the liquid (2900 bar) and crystal (3100 bar) phase. (b) SAXS profiles $I(q)$ at different times $t$ for the pressure jump to $p_{f}=3220$ bar averaged over 200 ms exposure time. Curves are shifted vertically for clarity. 
and 3100 bar are shown in Fig. 1 (a), suggesting the transition from the liquid to the crystal phase between both pressures. Exemplary SAXS profiles measured in a pressure jump series from $p_{0}=2700$ bar to $p_{f}=3220$ bar are shown in Fig. 1 (b). The scattering signal is dominated by the form factor of the gold core expressed by the oscillations with increasing $q$. This form factor does not show any variation with pressure 30 . With increasing $t$ after the pressure jump at $t=1 \mathrm{~s}$, a peak appears around $q=0.23$ $\mathrm{nm}^{-1}$, accompanied by modulations at larger $q$. As the form factor does not vary with $p$, we concentrate here on the structure factor $S(q)$ highlighting such modulations.

Fig. 2 shows the development of the structure factors $S(q)=$ $I(q) / P(q)$ for different pressure jumps starting at $p_{0}=2700$ bar and $p_{0}=2900$ bar, respectively, both below the crystallisation pressure $p_{c}$. At short times ( $t<1 \mathrm{~s}$ ) the structure factor is $S(q) \approx 1$, i.e., we do not observe any significant difference from a form factor as expected for the particle concentration used in this study. After the pressure jump at $t=1 \mathrm{~s}$, all measurements show similar trends. First, the intensity for $q \rightarrow 0 \mathrm{~nm}^{-1}$ grows, suggesting formation of agglomerates. Second, a peak appears around $q \approx 0.23$ $\mathrm{nm}^{-1}$ which is accompanied by modulations at larger $q$. Note that both features are not observed for $p_{f}<p_{c}$. While this observation proves the formation of ordered superstructures after the pressure jumps, the structure factor peaks appear faster and more pronounced the higher $p_{f}$. This suggests different kinetics of the superstructure nucleation and growth depending on $p_{f}$ and can be analyzed in more detail by tracking the peak value $S_{\max }$ of $S(q)$ over the experimental time $t$, see Fig. 3 (a). Therein, the results are shown from the four $S(q)$ series discussed in Fig. 2. For all $p_{f}$ the data shows strong fluctuations that resemble the statistical accuracy of the SAXS measurement due to the short exposure time. Nevertheless, an increase of $S_{\max }$ with $t$ is found for all $p_{f}$, indicating increasing degree of order in the system. For the two highest pressures shown $S_{\max }$ strongly increase after the pressure jump whereas the two lower pressures result in a slow increase. This was modeled by a limited exponential growth model as

$$
S_{\max }(t)-1=S_{\infty}-S_{\infty} \exp \left(-\frac{t-t_{j}}{\tau}\right),
$$

with $t_{j}=1 \mathrm{~s}$ as the time of the pressure jump. It is important to note that the fit was limited to the $t$-values for which the black lines were plotted in Fig. 3 (a). In general, $S_{\max }$ shows a second slower relaxation at longer times suggesting, e.g., that $S_{\max }$ for $p_{f}=3220$ bar will approach the value of $p_{f}=3580 \mathrm{bar}$ for $t \rightarrow \infty$.

As already visible from Fig. 2, the characteristic time $\tau$ decreases with $p_{f}$, i.e, the higher the pressure jump, the faster the formation of order in the sample system. The resulting growth limit $S_{\infty}$ is compared to the characteristic formation time $\tau$ in Fig. 3 (b). Therein results from an additional dataset with $p_{0}=2700$ bar and $p_{f}=2960$ bar very close to $p_{c}$ are shown as well. The characteristic time scale $\tau$ of the growth of $S_{\max }$ varies between $\tau(2960 \mathrm{bar}) \approx 25 \mathrm{~s}$ and $\tau(3580 \mathrm{bar}) \approx 0.3 \mathrm{~s}$ and roughly follows an exponential decay. The growth limit $S_{\infty}=S_{\max }(\tau \rightarrow \infty)$ increases from 1.14 to 2.55 . Obviously, the shorter the time scale $\tau$ to reach $S_{\infty}$ - and consequently the larger $\Delta p-$ the higher $S_{\infty}$ and thus the degree of order in the sample. We modeled this behavior by an exponential relation between $S_{\infty}$ and $\tau$ (solid line in Fig. 3 (b)) which suggests a limit of $S_{\infty}(\tau=0)=2.6$. However, due to the limited number of pressure jumps, other relations may describe the data as well fulfilling $S_{\infty}(\tau \rightarrow \infty) \rightarrow 1$.

In order to gain more insight into the structural variation after the pressure jump, we extracted the position $q_{\max }$ where $S\left(q_{\max }\right)=S_{\max }$ as well as the width $w$ of the first structure factor peaks shown in Fig. 2. The values have been obtained from Gaussian fits to the $S(q)$ peaks and are shown in Fig. 3(c). For $t<t_{j}=1$ $\mathrm{s}$ the data show a large spread because no significant peak can be found. Therefore, we concentrate the discussion to $t>t_{j}$. Depending on $p_{f}$, the first measurable values of $q_{\max }$ vary between $\sim 0.227 \mathrm{~nm}^{-1}$ and $\sim 0.235 \mathrm{~nm}^{-1}$. Furthermore, we found the higher $p_{f}$, the higher $q_{\max }$. With increase time $t, q_{\max }$ continues to grow and hardly saturates with our experimental time of $t_{\max }=30$ s. The position $q_{\max }$ can be associated to the particle-particle distance $d=2 \pi \sqrt{3 / 2} / q_{\max }$ assuming that it represents the position of the (111) fcc Bragg reflections. This assumption is supported by our previous publication 22 , where we studied the structure of the fcc superlattices formed at high pressures. The interparticle distance $d$ is also given on the right axis of Fig. 3 (c,top). Our results demonstrate, that $d$ shrinks by approximately 1 to $1.5 \mathrm{~nm}$ during the $29 \mathrm{~s}$ after the pressure jump depending of $p_{f}$, revealing as well a slow compression of the formed superstructures.

The peak width $w$ shown in Fig. 3 (c,bottom) exhibits a different behavior. While $w$ drops with $t$ with a rate that depends on $p_{f}$ - the higher $p_{f}$ the higher the rate - it saturates at different values $w_{f}$. The characteristic time $\tau_{w}$ and saturation width $w_{f}$ are modeled by an exponential assuming a constant value of $w_{0} \approx 0.6$ $\mathrm{nm}^{-1}$ for $t=t_{j}$ and are given in Tab. 1. In general, $\tau_{w}$ is found to be shorter than $\tau$. Thus, the distribution of interparticle distances is defined first, afterwards the number and size of crystallites grows (increase of $S_{\max }$ ). Furthermore, $w_{f}$ is higher for small $p_{f}$. Remarkably, the two highest studied pressure jumps do not differ significantly in $w_{f}$. As widths of Bragg reflections are typically reciprocally connected to larger superstructures with higher crystallinity, we conclude that the quality of the superstructure formed by pressure jumps can be tuned by $p_{f}$.

Table 1 Characteristic time $\tau_{w}$ and saturation width $w_{f}$ of the peak width for different pressure jumps.

\begin{tabular}{lll}
\hline pressure $p_{f}$ (bar) & time $\tau_{w}(\mathrm{~s})$ & width $w_{f}\left(\mathrm{~nm}^{-1}\right)$ \\
\hline 3040 & $6.1 \pm 0.9$ & $0.138 \pm 0.013$ \\
3130 & $3.1 \pm 0.2$ & $0.078 \pm 0.002$ \\
3220 & $0.51 \pm 0.02$ & $0.0492 \pm 0.0006$ \\
3580 & $0.07 \pm 0.02$ & $0.0458 \pm 0.0003$ \\
\hline
\end{tabular}

As reported earlier 22 , the superstructures were found to melt once the pressure dropped to 1 bar. To study the kinetics of this melting process, we performed a pressure jump from 3220 bar to 2770 bar. The sample shown in Fig. 2 (bottom, left) was kept at 3220 bar for few minutes to allow the crystal to grow. Afterwards, the pressure jump protocol was used in the same way as before, i.e., at $t=1 \mathrm{~s}$ of the SAXS pattern series the pressure jump to 2770 bar has been performed, see Fig. 4 (a). Consequently, the structure factors measured at $t<1 \mathrm{~s}$ show a narrower and asym- 


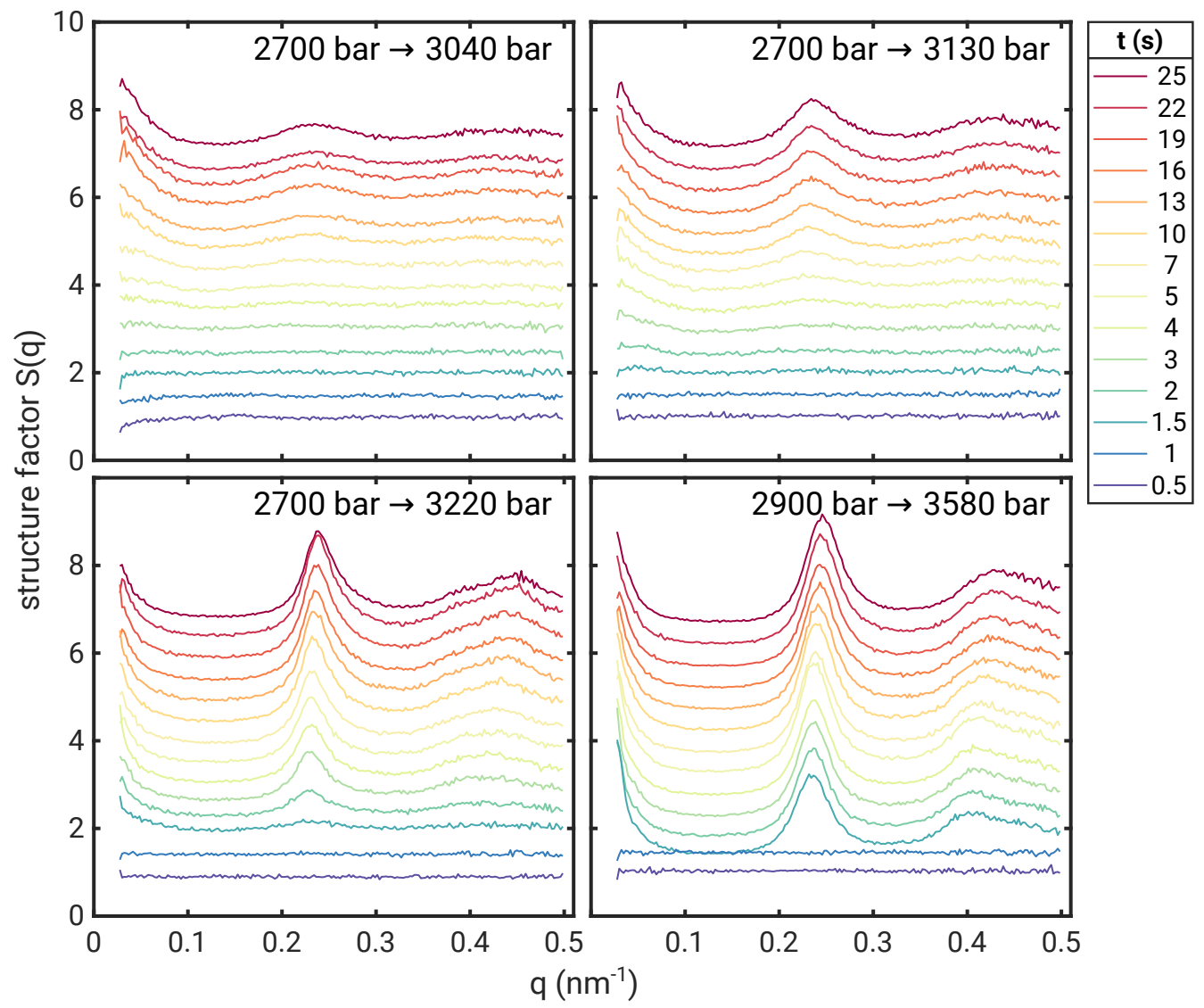

Fig. 2 Structure factor $S(q)$ variation for $q<0.5 \mathrm{~nm}^{-1}$ after the pressure jumps. The pressure jump took place at $t \approx 1 \mathrm{~s}$. The initial and final pressure $p_{0}$ and $p_{f}$ are labeled in each subfigure. The curves are shifted vertically for clarity, successively by 0.5 .
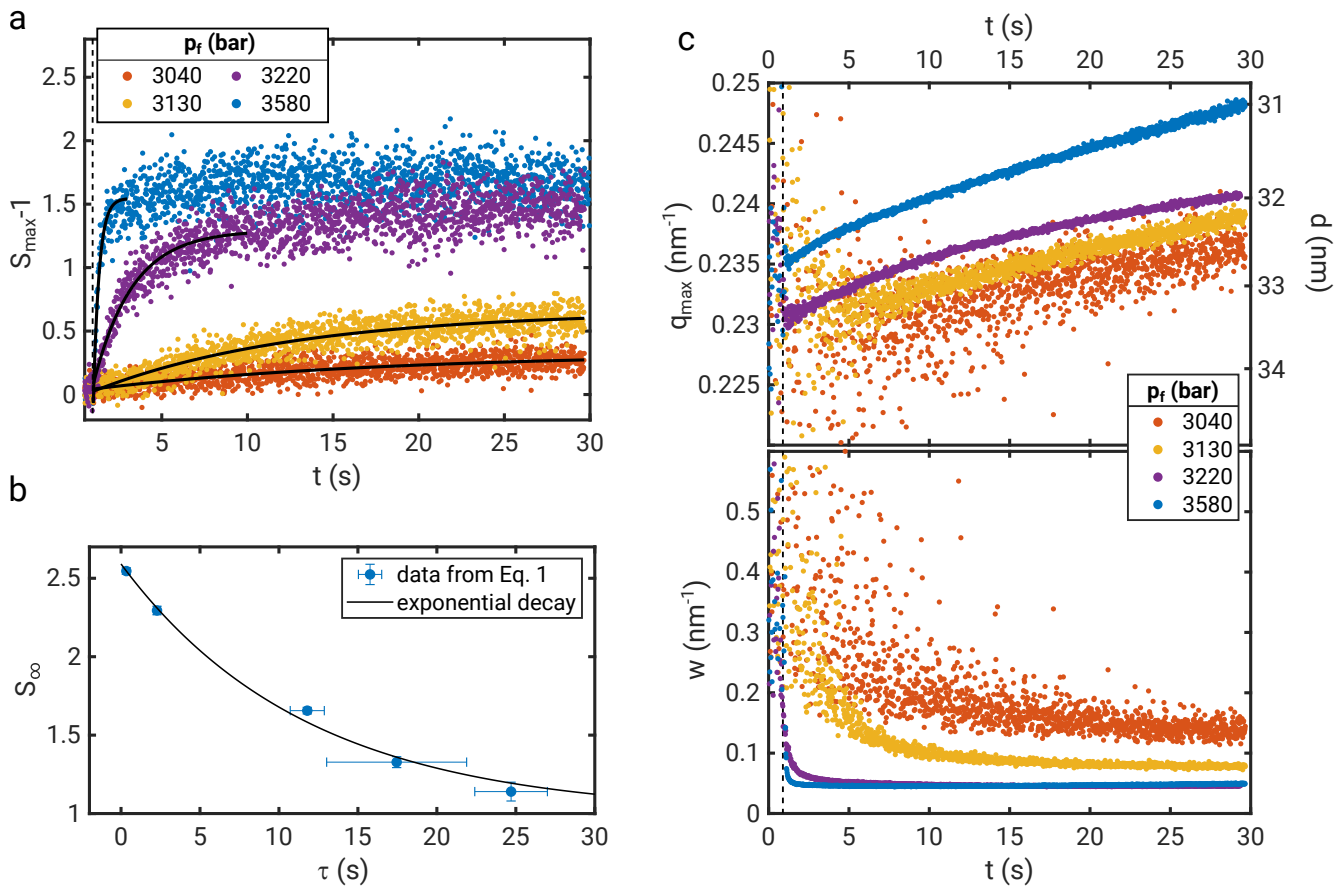

Fig. 3 (a) Structure factor peak value $S_{\max }$ as function of $t$. The dashed line marks the pressure jump at $t \approx 1 \mathrm{~s}$. Solid lines are fits of Eq. 1. (b) Growth limit $S_{\infty}$ as function of time scale $\tau$ from fits shown in panel (a) for part of the data. Solid line is an exponential decay as guide to the eye. (c) Position $q_{\max }$ (top) and width $w$ (bottom) of the first $S(q)$ peak. The dashed line marks the pressure jump at $t \approx 1 \mathrm{~s}$. 

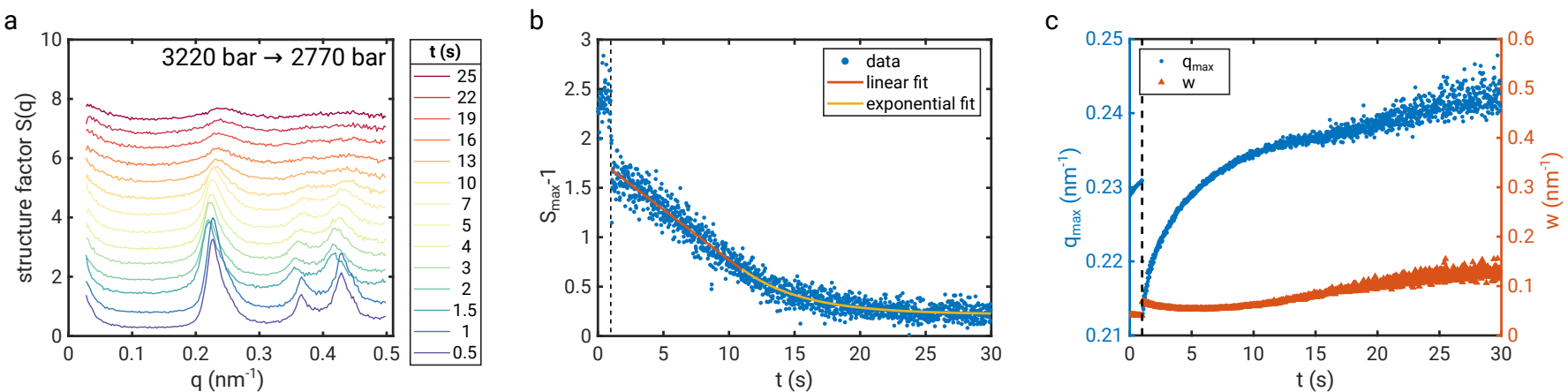

Fig. 4 Kinetics of pressure release. (a) Structure factors $S(q)$ before and after pressure release from 3220 bar to 2770 bar. (b) Structure factor peak value $S_{\max }$ variation after pressure release at $t_{j}=1 \mathrm{~s}$ (dashed line). (c) Evolution of peak position $q_{\max }$ and peak width $w$ after the pressure release (dashed line).

metric structure factor peak around $q_{\max } \approx 0.22 \mathrm{~nm}^{-1}$ together with well-developed peaks at larger $q$. This resembles well the structure factor of a fcc lattice, covering the (111) reflection at $q_{\max }$ with the (200) as shoulder at $0.25 \mathrm{~nm}^{-1}$ as well as the (220) and (311) reflection at $q_{220} \approx 0.37 \mathrm{~nm}^{-1}$ and $q_{311} \approx 0.43 \mathrm{~nm}^{-1}$, respectively. After the pressure jump at $t_{j}=1 \mathrm{~s}$, the peaks become less pronounced. This is demonstrated by tracking $S_{\max }$ as a function of $t$, see Fig. 4 (b). Remarkably, at $t_{j} S_{\max }$ suddenly drops from approximately 3.4 to 2.6 , which resembles the maximum observed $S_{\infty}$ in Fig. 3 (b). Afterwards, we observe a linear drop of $S_{\max }$ with a rate of $\Delta S_{\max }=-0.10 \mathrm{~s}^{-1}$, which levels off for $t>10$ s, empirically modeled by an exponential decay. In Fig. 4 (c) the variation of the corresponding $q_{\max }$ and $w$ are shown. At $t_{j}$, both rapidly drop similar to $S_{\max }$. The drop of $q_{\max }$ and the increase of $w$ reflect a sudden increase of the lattice constant with a reduced crystallinity due to swelling of the PEG shells. Afterwards, $w$ relaxes slightly, before it continues to grow as expected for crystal melting. In contrast, $q_{\max }$ grows as well suggesting further compression. However, the remaining peak is very weak (cf. Fig. 4 a) which we interpret as a fingerprint from remaining agglomerates with less defined inter-particle distance. Such agglomerates were found to disappear after further waiting 22 .

\section{Discussion}

We have studied the kinetics of superlattice formation following pressure jumps. The crystallisation pressure for our PEGylated AuNC system at $2 \mathrm{M} \mathrm{RbCl}$ resembled the values reported earlier ${ }^{22}$. We associate the difference between the structure factors reported here and in ref. ${ }^{22}$, namely the smaller number of Bragg reflections visible, mainly to the different experimental protocol. In our previous study the pressure was increased significantly slower. Furthermore, we added waiting times allowing the crystal to grow as represented by a high number of Bragg reflections. As we focused on the formation kinetics in this study we chose short exposure times which challenges a more detailed analysis of Bragg reflections as performed in ${ }^{22}$. By tracking the evolution of the structure factors after pressure quenches above the crystallisation pressure, we were able to follow the superlattice growth with few ms time resolution. First, the peak of the structure factors representing the superlattice growth was found to follow an exponential and saturates at a certain value $S_{\infty}$. The higher the pressure jump $\Delta p$, the faster the superlattice formation.

This can be rationalized with a change of particle-particle interaction at higher pressure. With increasing pressure, the interaction potential becomes attractive ${ }^{30 \mid 31}$. When the critical pressure is reached, the solubility of the PEGylated AuNC is reduced ${ }^{22}$ and water is expelled from the PEG shell ${ }^{32}$, similar to temperature changes in thermoresponsive gels ${ }^{33}$. This results in an increasing attractive interaction dominated by the less hydrated and less swollen PEG shells. Furthermore, it supports our interpretation of the superlattice formation as a phase transition that can be attributed to the solubility properties of the PEG shells ${ }^{22}$, which exhibit - analogue to the lower critical solution temperature LCST - a lower critical solution pressure LCSP, that is, the critical pressures reported herein. The larger the pressure jump, the more attractive the interaction. Consequently, the superlattice forms faster because of higher probability of particles to interact and bind ${ }^{34}$. Although the interparticle interaction is attractive, the PEG-shells prevent sintering. The fcc structure of the crystallites indicates that the attractive interactions are weak, allowing for some reorganisation of the assembled NC. In contrast, a strong interparticle binding would favor fractal assemblies without fcc structure 34 .

Second, the faster the superlattice grows (small $\tau$ ), the better the crystal quality, expressed by the value of $S_{\infty}$ and the width of the structure factor peaks $w$. Furthermore, the superlattices were found to continuously compress after the pressure jump, showing a reduction of next-neighbour distance of about $1 \mathrm{~nm}$ within 30 $\mathrm{s}$ after the pressure jump. Third, while the superlattice formation was found to be exponential, melting after fast pressure release was found to follow linear kinetics and happens approximately one order of magnitude slower than the superlattice formation.

Our results indicate that modification of $\Delta p$ allows tuning the crystalline quality of the assembled superlattice. In particular, fine-tuning of the next-neighbour distance is possible withing fraction of the particle size. This paves the way towards preparation of high-quality NC assemblies directly from concentrated nanocrystal solutions by "freezing" the NC assembly at a certain selection of the width of pressure jump $\Delta p$ and time $t$. It should also be possible to reversibly "capture" analytes within the superlattices for enhanced spectroscopy studies and applications. Our study demonstrates that pressure allows for extremely fast for- 
mation of high quality plasmonic superlattices, and could therefore help producing defined superlattices in various matrices (e.g. polymer melts). Future studies are encouraged to understand the role of this parameter in superlattice synthesis and modification and to explore possible applications.

\section{Conflicts of interest}

There are no conflicts to declare.

\section{Acknowledgements}

The authors thank Andrew J. Smith, Tim Snow, and Nick Terrill for support during the experiment at I22 at Diamond Light Source under proposal SM18875. This work is supported by the Cluster of Excellence 'Advanced Imaging of Matter' of the Deutsche Forschungsgemeinschaft (DFG) - EXC 2056 - project ID 390715994. Florian Schulz acknowledges the Deutsche Forschungsgemeinschaft (grant No. SCHU 3019/2-1), Martin A. Schroer acknowledges the Röntgen-Ångström cluster project TTSAS (Bundesministerium für Bildung und Forschung project No. 05K16YEA) for financial support.

\section{Notes and references}

1 D. J. Milliron, R. Buonsanti, A. Llordes and B. A. Helms, Acc. Chem. Res., 2013, 47, 236-246.

2 F. Lu, K. G. Yager, Y. Zhang, H. Xin and O. Gang, Nat. Commun., 2015, 6, 6912.

3 M. V. Kovalenko, L. Manna, A. Cabot, Z. Hens, D. V. Talapin, C. R. Kagan, V. I. Klimov, A. L. Rogach, P. Reiss, D. J. Milliron, P. Guyot-Sionnnest, G. Konstantatos, W. J. Parak, T. Hyeon, B. A. Korgel, C. B. Murray and W. Heiss, ACS Nano, 2015, 9, 1012-1057.

4 C. R. Kagan, E. Lifshitz, E. H. Sargent and D. V. Talapin, Science, 2016, 353, aac5523.

5 C. Hanske, E. H. Hill, D. Vila-Liarte, G. González-Rubio, C. Matricardi, A. Mihi and L. M. Liz-Marzán, ACS Applied Materials Interfaces, 2019, 11, 11763-11771.

6 W. Wei, F. Bai and H. Fan, Angew. Chem. Int. Ed., 2019, 58, doi:10.1002/anie.201902620.

7 D. García-Lojo, S. Núñez-Sánchez, S. Gómez-Graña, M. Grzelczak, I. PastorizaSantos, J. Pérez-Juste and L. M. Liz-Marzán, Acc. Chem. Res., 2019, 52, doi:10.1021/acs.accounts.9b00213.

8 C. B. Murray, C. R. Kagan and M. G. Bawendi, Science, 1995, 270, 1335-1338.

9 K. J. M. Bishop, C. E. Wilmer, S. Soh and B. A. Grzybowski, Small, 2009, 5, 1600-1630.

10 N. Vogel, M. Retsch, C.-A. Fustin, A. del Campo and U. Jonas, Chem. Rev., 2015, 115, 6265-6311.

11 M. A. Boles, M. Engel and D. V. Talapin, Chem. Rev., 2016, 116, 11220-11289.

12 J. Gong, R. S. Newman, M. Engel, M. Zhao, F. Bian, S. C. Glotzer and Z. Tang, Nat. Commun., 2017, 8, 14038.

13 S. Connolly, S. Fullam, B. Korgel and D. Fitzmaurice, J. Am. Chem. Soc., 1998, 120, 2969-2970.

14 S. Narayanan, J. Wang and X.-M. Lin, Phys. Rev. Lett., 2004, 93, 135503.

15 Y. Yu, A. Jain, A. Guillaussier, V. R. Voggu, T. M. Truskett, D.-M. Smilgies and B. A. Korgel, Faraday Discussions, 2015, 181, 181-192.

16 Y. Yu, A. Guillaussier, V. R. Voggu, W. Pineros, T. M. Truskett, D.-M. Smilgies and B. A. Korgel, J. Phys. Chem. C, 2016, 120, 27682-27687.

17 T. Li, A. J. Senesi and B. Lee, Chem. Rev., 2016, 116, 11128-11180.

18 Y. Liu, M. Agthe, M. Salajková, K. Gordeyeva, V. Guccini, A. Fall, G. SalazarAlvarez, C. Schütz and L. Bergström, Nanoscale, 2018, 10, 18113-18118.

19 M. C. Weidman, D.-M. Smilgies and W. A. Tisdale, Nat. Mater., 2016, 15, 775781.

20 Y. Yu, D. Yu, B. Sadigh and C. A. Orme, Nat. Commun., 2018, 9, 4211.

21 I. Lokteva, M. Koof, M. Walther, G. Grübel and F. Lehmkühler, Small, 2019, 15, 1900438.

22 M. A. Schroer, F. Lehmkühler, J. Möller, H. Lange, G. Grübel and F. Schulz, J. Phys. Chem. Lett., 2018, 9, 4720-4724.

23 F. Bai, K. Bian, X. Huang, Z. Wang and H. Fan, Chem. Rev., 2019, 119, 76737717.

24 F. Schulz, T. Vossmeyer, N. G. Bastús and H. Weller, Langmuir, 2013, 29, 98979908.

25 F. Schulz, G. T. Dahl, S. Besztejan, M. A. Schroer, F. Lehmkühler, G. Grübel, T. Vossmeyer and H. Lange, Langmuir, 2016, 32, 7897-7907.

26 F. Lehmkühler, F. Schulz, M. A. Schroer, L. Frenzel, H. Lange and G. Grübel, IUCrJ, 2018, 5, 354-360.
27 F. Lehmkühler, F. Schulz, M. A. Schroer, L. Frenzel, H. Lange and G. Grübel, $J$ Appl. Cryst., 2019, 52, 777-782.

28 A. J. Smith, L. S. Davidson, J. H. Emmins, J. C. H. Bardsley, P. Holloway, M. Mal fois, A. R. Marshall, C. L. Pizzey, S. E. Rogers, O. Shebanova, T. Snow, E. P. Williams and N. J. Terrill, arXiv, 2019, 1903.05405.

29 N. J. Brooks, B. L. L. E. Gauthe, N. J. Terrill, S. E. Rogers, R. H. Templer, O. Ces and J. M. Seddon, Rev. Sci. Instrum., 2010, 81, 064103.

30 M. A. Schroer, F. Schulz, F. Lehmkühler, J. Möller, A. J. Smith, H. Lange, T. Vossmeyer and G. Grübel, J. Phys. Chem. C, 2016, 120, 19856-19861.

31 H. Wu, Z. Wang and H. Fan, J. Am. Chem. Soc., 2014, 136, 7634-7636.

32 F. Schulz, J. Möller, F. Lehmkühler, A. J. Smith, T. Vossmeyer, H. Lange, G. Grübel and M. A. Schroer, Part. Part. Sys. Charact., 2017, 35, 1700319.

33 M. Das, H. Zhang and E. Kumacheva, Annu. Rev. Mater. Res., 2006, 36, 117-142.

34 G. Odriozola, A. Moncho-Jordá, A. Schmitt, J. Callejas-Fernández, R. MartínezGarcía and R. Hidalgo-Álvarez, Europhys. Lett., 2001, 53, 797-803. 